\title{
Matroid And Its Outlines
}

\author{
Henry Garrett \\ Independent Researcher \\ DrHenryGarrett@gmail.com \\ Twitter's ID: @DrHenryGarrett | CB08PDK8J5G
}

\begin{abstract}
In this article, there's an effort to make sense about the new versions of matroid. I believe that there's new idea on the background of. matroid. Two styles of matroid is defined in the background of fixed graphs and after that the attributes of these new notion on the graph and its parameters have been studied. The focus of this article is on the version of matroid which has the basis on the cycles as if there's gentle discussion on the results which are based on the set of independent vertices as matroid-x. The relation amid fundamental parameters and specific set like independent set and minimal set in the terminology of graph theory have been considered. Matroid is the word to use in the study on the parameters of graph theory as if set theory and its terminology are also recorded. The terms of word in various terminology have been relatively used.

There are open ways to use hypergraphs or some serious relations amid these two types.
\end{abstract}

Keywords: Graph theory, Complete graph, Independent set, Power set.

AMS Subject Classification: 05C17, 05C22, 05E45, 05E14

\section{Preliminary On The Concept}

I'm going to refer to some books which are cited to the necessary and sufficient material which are covering the introduction and the preliminary of this outlet so look [Ref. [1], Ref. [2], Ref. [3], Ref. [4]] where Ref. [1] is about the textbook, Ref. [2] is common, Ref. [3] has good ideas and Ref. [4] is kind of disciplinary approaches in the good ways. Further references could be referred and could be addressed in Refs. [5-11].

\section{Definition And Its Clarification}

The kind of numbers have been used to differentiate amid vertices. There's the same condition for edges so $\{1,2,3,4,5,6\}$ is the set of vertices and the set $\{1,2,3,4,5,6\}$ is the set of edges. Let $\mathcal{B}$ is the set of subsets of $\mathcal{E}$ which are cycles so as an example, $\{\{1\},\{2,3\},\{4,5,6\}\}$ where the singleton is the loop and set with couple of numbers is the parallel cycles. To capture the details, the upcoming paragraph is up.

Definition 2.1. (Matroid)

Let $\mathcal{G}:(\mathcal{V}, \mathcal{E})$ be a graph. Let $\mathcal{B}$ be the set of some cycles. If there's three conditions for these two sets, then there's matroid which is corresponded to $(\mathcal{E}, \mathcal{B})$. So $(\mathcal{E}, \mathcal{B})$ is the characteristic of the matroid. Three conditions are the following: 
- The set which has no member, belongs to $B$.

- If $b \in B$, then any of subset of $b$, belongs to $B$.

- If $b_{1}, b_{2} \in B$ and $b_{1}$ has more members than $b_{2}$ then there's the member of $b_{1}$, when it's added to $b_{2}$, it makes the new member of $B$.

\section{Relationships And Its illustrations}

Theorem 3.1. Let $(\mathcal{E}, \mathcal{P}(\mathcal{E}))$ be a matroid. Then

(i) $\operatorname{Size}(\mathcal{G})=C(n, 2)+2 n$;

(ii) $\operatorname{Order}(\mathcal{G})=\log _{2}^{|\mathcal{B}|}$;

(iii) $|\mathcal{B}|=2^{n}$;

(iv) $\Delta(G)=\delta(\mathcal{G})=n$;

(v) Background's graph is complete;

(vi) $\operatorname{Order}(\mathcal{G})$ is $\operatorname{Size}(\mathcal{G})-C(n, 2)$ half.

Proof. Obvious.

\section{Results And Its Beyond}

Theorem 4.1. Let $(\mathcal{E}, \mathcal{B})$ be a matroid. Then

(i) $\operatorname{Size}(\mathcal{G}) \leq C(n, 2)+2 n$;

(ii) $\operatorname{Order}(\mathcal{G}) \leq \log _{2}^{|\mathcal{B}|}$;

(iii) $|\mathcal{B}| \leq 2^{n}$;

(iv) $\Delta(\mathcal{G}) \leq n ;$

(v) $\delta(\mathcal{G}) \leq n$

(vi) $\operatorname{Order}(\mathcal{G})$ is lower than $\operatorname{Size}(\mathcal{G})-C(n, 2)$ half.

Proof. Obvious.

Theorem 4.2. Let $(\mathcal{E}, \mathcal{B})$ be a matroid and $B$ has one member. Then Background's graph is empty.

Proof. Obvious.

Theorem 4.3. Let $(\mathcal{E}, \mathcal{B})$ be a matroid. Then $1 \leq|\mathcal{B}| \leq 2^{n}$.

Proof. Obvious.

Theorem 4.4. Let $(\mathcal{E}, \mathcal{B})$ be a matroid. If $\mathcal{B}=\left\{b_{i} \mid i=1,2, \cdots, n\right\}$ then $\left\{\mathcal{P}\left(b_{1}\right), \mathcal{P}\left(b_{2}\right), \cdots, \mathcal{P}\left(b_{n}\right)\right\} \subseteq \mathcal{B}$.

Proof. Obvious.

Theorem 4.5. Let $(\mathcal{E}, \mathcal{B})$ be a matroid. If $b_{1}, b_{2} \in \mathcal{B}$ such that $\left|b_{1}\right|<\left|b_{2}\right|$ then

- $\mathcal{P}\left(b_{1}\right)<\mathcal{P}\left(b_{2}\right)$; 
- $\mathcal{P}\left(b_{1}\right), \mathcal{P}\left(b_{2}\right) \in \mathcal{B}$;

- there's $e \in b_{2}-b_{1}$ such that $\mathcal{P}\left(b_{1} \cup\{e\}\right), \mathcal{P}\left(b_{2}\right) \in \mathcal{B}$;

- $\mathcal{P}\left(b_{1}\right) \cup \mathcal{P}\left(b_{2}\right) \in \mathcal{B}$;

- if $b_{1} \subseteq b_{2}$, then $b_{1} \in \mathcal{P}\left(b_{2}\right)$;

- if $b_{1} \cup b_{2}=\mathcal{E}$, then $\mathcal{B}=\mathcal{P}(\mathcal{E})$.

Proof. Obvious.

Theorem 4.6. Let $(\mathcal{E}, \mathcal{B})$ be a matroid. If all members of $\mathcal{B}$ are singleton if and only if $|\mathcal{B}|$ equals order of $\mathcal{G}$.

Proof. Obvious.

Theorem 4.7. Let $(\mathcal{E}, \mathcal{B})$ be a matroid. If all members of $\mathcal{B}$ are singleton. and $|\mathcal{B}|$ equals order of $\mathcal{G}$. Then $\mathcal{G}$ is neither complete graph nor $\mathcal{B}=\mathcal{P}(\mathcal{G})$.

Proof. Obvious.

Theorem 4.8. Let $(\mathcal{E}, \mathcal{B})$ be a matroid. If $\forall b \in \mathcal{B},|b| \leq 2$. Then $|\mathcal{B}|$ equals $2 C(\operatorname{Order}(\mathcal{G}), 2)+\operatorname{Order}(\mathcal{G})$.

Proof. Obvious.

Theorem 4.9. Let $(\mathcal{E}, \mathcal{B})$ be a matroid. If all members of $\mathcal{B},|b| \leq 2$. And $|\mathcal{B}|$ equals $2 C(\operatorname{order}(\mathcal{G}), 2)+\operatorname{order}(\mathcal{G})$. Then $\mathcal{G}$ is neither complete graph nor $\mathcal{B}=\mathcal{P}(\mathcal{G})$.

Proof. Obvious.

Theorem 4.10. Let $(\mathcal{E}, \mathcal{B})$ be a matroid. If there's $b \in \mathcal{B}$ such that $|b|=c$. Then $\mathcal{G}$ has a complete subgraph from the order $c$.

Proof. Obvious.

Theorem 4.11. Let $(\mathcal{E}, \mathcal{B})$ be a matroid. If there's $b \in \mathcal{B}$ such that $|b|=\operatorname{Order}(\mathcal{G})$. Then $\mathcal{G}$ is a complete graph.

Proof. Obvious.

Theorem 4.12. Let $(\mathcal{E}, \mathcal{B})$ be a matroid. If there's $b \in \mathcal{B}$ such that $|b|=\operatorname{Order}(\mathcal{G})$. Then $\mathcal{B}=\mathcal{P}(\mathcal{E})$.

Proof. Obvious.

Theorem 4.13. Let $(\mathcal{E}, \mathcal{B})$ be a matroid. If for all $b \in \mathcal{B},|b|=2$ or 1 and $\mathcal{B}$ has two partitions. Then $\mathcal{G}$ has a complete bipartite graph.

Proof. Obvious.

Theorem 4.14. Let $(\mathcal{E}, \mathcal{B})$ be a matroid. If there's $b \in \mathcal{B},|b|>1$. Then $b$ isn't a minimal sets in the terms of having membership of $\mathcal{B}$.

Proof. Obvious.

Theorem 4.15. Let $(\mathcal{E}, \mathcal{B})$ be a matroid. If there's $b \in \mathcal{B},|b|>1$. Then subsets of $b$ which are singleton, are the minimal sets in the terms of having membership of $\mathcal{B}$.

Proof. Obvious. 
Definition 4.16. (Matroid-x)

Let $\mathcal{G}:(\mathcal{V}, \mathcal{E})$ be a graph. Let $\mathcal{B}$ be the set of some sets of independent vertices. If there's three conditions for these two sets, then there's matroid-x which is corresponded to $(\mathcal{V}, \mathcal{B})$. So $(\mathcal{V}, \mathcal{B})$ is the characteristic of the matroid-x. Three conditions are the following:

- The set which has no member, belongs to $B$.

- If $b \in B$, then any of subset of $b$, belongs to $B$.

- If $b_{1}, b_{2} \in B$ and $b_{1}$ has more members than $b_{2}$ then there's the member of $b_{1}$, when it's added to $b_{2}$, it makes the new member of $B$.

Theorem 4.17. Let $(\mathcal{V}, \mathcal{B})$ be a matroid-x. If there's $b \in \mathcal{B},|b|>1$. Then subsets of $b$ which are singleton, are the minimal sets in the terms of having membership of $\mathcal{B}$.

Proof. Obvious.

Theorem 4.18. Let $(\mathcal{V}, \mathcal{B})$ be a matroid-x. If there's $b \in \mathcal{B},|b|>1$. Then $b$ isn't a minimal sets in the terms of having membership of $\mathcal{B}$.

Proof. Obvious.

Theorem 4.19. Let $\mathcal{G}:(\mathcal{V}, \mathcal{E})$ be a graph. Consider $\mathcal{V} \in \mathcal{B}$. If $(\mathcal{V}, \mathcal{B})$ is a matroid-x, then $\left(\mathcal{E}, \mathcal{B}^{\prime}\right)$ isn't a matroid.

Proof. Obvious.

Theorem 4.20. Let $\mathcal{G}:(\mathcal{V}, \mathcal{E})$ be a graph. Consider $\mathcal{E} \in \mathcal{B}$. If $(\mathcal{E}, \mathcal{B})$ is a matroid-x, then $\left(\mathcal{V}, \mathcal{B}^{\prime}\right)$ isn't a matroid.

Proof. Obvious.

Theorem 4.21. Let $(\mathcal{E}, \mathcal{B})$ be a matroid. Then the following statements are equiavalent:

- $\mathcal{E} \in \mathcal{B}$

- $\mathcal{P}(\mathcal{E})=\mathcal{B}$

- $\mathcal{G}:(\mathcal{V}, \mathcal{E})$ is complete graph.

Proof. Obvious.

Theorem 4.22. Let $(\mathcal{V}, \mathcal{B})$ be a matroid-x. Then the following statements are equiavalent:

- $\mathcal{V} \in \mathcal{B}$

- $\mathcal{P}(\mathcal{V})=\mathcal{B}$

- $\mathcal{G}:(\mathcal{V}, \mathcal{E})$ is empty graph.

Proof. Obvious.

Theorem 4.23. Let $\mathcal{G}:(\mathcal{V}, \mathcal{E})$ be a graph. Consider $\mathcal{E} \in \mathcal{B}$. If $(\mathcal{E}, \mathcal{B})$ is a matroid, then $\left(\mathcal{V}, \mathcal{B}^{\prime}\right)$ isn't a matroid- $x$.

Proof. Obvious.

Theorem 4.24. Let $\mathcal{G}:(\mathcal{V}, \mathcal{E})$ be a graph. If $\mathcal{I}$ is an independent set of vertices in the term of having edges amid them, then $\mathcal{B}=\mathcal{I}$ and $(\mathcal{V}, \mathcal{B})$ is a matroid-x where singleton $b \in \mathcal{I}$ is considered as $\{b\}$ in the set $\mathcal{B}$ or $\mathcal{I} \in \mathcal{B}$. 
Proof. Obvious.

Theorem 4.25. Let $\mathcal{G}:(\mathcal{V}, \mathcal{E})$ be a graph. If $\mathcal{M}$ is a minimal set of cycles in the term of having membership of $\mathcal{B}$, then either $\mathcal{B}=\mathcal{M}$ and $(\mathcal{V}, \mathcal{B})$ is a matroid-x where singleton $b \in \mathcal{M}$ is considered as $\{b\}$ in the set $\mathcal{B}$ or $\mathcal{M} \notin \mathcal{B}$.

Proof. Obvious.

\section{References}

1. R. Balakrishnan, K. Ranganathan, A Textbook of Graph Theory, New York, 2012.

2. Adrian Bondy, U.S.R Murty, Graph Theory, New York, 2008.

3. Michael Capobianco, and John C. Molluzzo, Examples and counterexamples in graph theory, New York, 1978.

4. Chris Godsil, and Gordon Royle, Algebraic Graph Theory, New York, 2001.

5. Joseph P.S. Kung, Inconsequential results on the Merino-Welsh conjecture for Tutte polynomials. Preprint available at https://arxiv.org/abs/2105.01825.

6. Duksang Lee, $\Gamma$-graphic delta-matroids and its applications. Preprint available at https://arxiv.org/abs/2104.11383.

7. Luis Ferroni, Matroids are not Ehrhart positive. Preprint available at https://arxiv.org/abs/2105.04465.

8. Luis Ferroni, Lorenzo Vecchi, Matroid relaxations and Kazhdan-Lusztig non-degeneracy. Preprint available at https://arxiv.org/abs/2104.14531.

9. Norbert Peyerimhoff, Marc Roth, Johannes Schmitt, Jakob Stix, Alina Vdovina, Parameterized (Modular) Counting and Cayley Graph Expanders. Preprint available at https://arxiv.org/abs/2104.14596.

10. James Oxley, Zach Walsh, 2-Modular Matrices. Preprint available at https://arxiv.org/abs/2105.04525.

11. Zach Walsh, New lift matroids for group-labeled graphs. Preprint available at https://arxiv.org/abs/2104.08257. 